\begin{tabular}{|c|c|}
\hline Title & $\begin{array}{l}\text { Linear Correlations between A dsorption Energies and HOMO Levels for the A dsorption of Small Molecules on TiO2 } \\
\text { Surfaces }\end{array}$ \\
\hline Author(s) & $\begin{array}{l}\text { Kamachi, Takashi; Tatsumi, Toshinobu; Toyao, T akashi; Hinuma, Y oyo; Maeno, Zen; Takakusagi, Satoru; Furukawa, } \\
\text { Shinya; T Takigawa, Ichigaku; Shimizu, Ken-ichi }\end{array}$ \\
\hline Citation & $\begin{array}{l}\text { Journal of phy sical chemistry c, } 123(34), 20988-20997 \\
\text { https://doi.org/10.1021/acs.jpcc.9b05707 }\end{array}$ \\
\hline Issue Date & $2019-08-29$ \\
\hline Doc URL & http:/hdl.handle.net/2115/79144 \\
\hline Rights & $\begin{array}{l}\text { This document is the A ccepted Manuscript version of a Published Work that appeared in final form in Journal of } \\
\text { Phy sical Chemistry C, copyright c A merican Chemical Society after peer review and technical editing by the publisher. } \\
\text { To access the final edited and published work see https://pubs.acs.org/doi/10.1021/acs.jpcc.9b05707. }\end{array}$ \\
\hline Type & article (author version) \\
\hline File Information & Text190801-submit.pdf \\
\hline
\end{tabular}

Instructions for use 


\section{Linear Correlations between Adsorption Energies and HOMO Levels for the Adsorption of Small Molecules on $\mathrm{TiO}_{2}$}

\section{Surfaces}

Takashi Kamachi*,1,3 Toshinobu Tatsumi, ${ }^{1}$ Takashi Toyao, ${ }^{2,3}$ Yoyo Hinuma, ${ }^{4,5}$ Zen Maeno, ${ }^{2}$ Satoru Takakusagi, ${ }^{2}$ Shinya Furukawa, ${ }^{2,3}$ Ichigaku Takigawa, ${ }^{6,7}$ and Ken-ichi Shimizu ${ }^{2,3}$

${ }^{1}$ Department of Life, Environment and Applied Chemistry, Fukuoka Institute of Technology (FIT), 3-30-1 Wajiro-Higashi, Higashi-ku, Fukuoka 811-0295, Japan ${ }^{2}$ Institute for Catalysis, Hokkaido University, N-21, W-10, Sapporo, 001-0021, Japan ${ }^{3}$ Elements Strategy Initiative for Catalysts \& Batteries, Kyoto University, Katsura, Kyoto 615-8520, Japan

${ }^{4}$ Center for Frontier Science, Chiba University, 1-33 Yayoicho, Inage, Chiba 263-8522, Japan

${ }^{5}$ Center for Materials Research by Information Integration, Research and Services Division of Materials Data and Integrated System, National Institute for Materials Science, 1-2-1 Sengen, Tsukuba, Ibaraki 305-0047, Japan

${ }^{6}$ RIKEN Center for Advanced Intelligence Project, 1-4-1 Nihonbashi, Chuo-ku, Tokyo 103-0027, Japan

${ }^{7}$ Institute for Chemical Reaction Design and Discovery (WPI-ICReDD), Hokkaido University Kita 21 Nishi 10, Kita-ku, Sapporo, Hokkaido 001-0021, Japan

Received xxxxx, 2019

*Author to whom correspondence should be addressed.

Tel: $+81-92-606-3867$

kamachi@fit.ac.jp (T.K.) 


\section{Abstract}

Adsorption is a fundamental step in catalysis on a solid surface, and therefore its understanding is important to explaining its behavior. This work investigated the adsorption of various small molecules, including $\mathrm{H}_{2}, \mathrm{~N}_{2}, \mathrm{CO}, \mathrm{CO}_{2}, \mathrm{CH}_{4}, \mathrm{NH}_{3}, \mathrm{H}_{2} \mathrm{O}, \mathrm{H}_{2} \mathrm{~S}$, DMSO, alkanes, alkenes, alkynes, aromatic compounds, alcohols, aldehydes, ketones, nitriles, carboxylic acids, amides, and amines, on the anatase (101) and rutile (110) surfaces of $\mathrm{TiO}_{2}$, using periodic density functional theory calculations and statistical methods. Adsorption energies were computed at the same level of theory to obtain a clean and consistent data set. A linear relationship was observed between the adsorption energies of these molecules and their highest occupied molecular orbital (HOMO) levels, whereas no obvious correlation was evident for the lowest unoccupied molecular orbital (LUMO) levels. Improved correlations between the adsorption energies and the HOMO levels were generated by dividing these molecules into two subgroups: hydrocarbons and heteroatom-containing compounds. Interactions between frontier molecular orbitals and the surfaces were considered, to gain a better understanding of the significant correlations that were identified. The data show that these relationships can be primarily ascribed to interactions between the HOMO of the small molecule and conduction state of the $\mathrm{TiO}_{2}$ surface. Statistical analysis using machine learning demonstrated that the HOMO and the dipole moment are the first and second most important properties, respectively, in terms of rationalizing and predicting the adsorption energies. 


\section{Introduction}

Since its development in the early 1950s, frontier molecular orbital (FMO) theory has provided a basis for explaining many aspects of chemical reactivity. ${ }^{1-2}$ According to Fukui's FMO theory ${ }^{3}$ and the Woodward-Hoffmann rule, ${ }^{4-7}$ the selectivity of organic reactions is determined by both phase properties and the magnitudes of the coefficients of the reactant's highest occupied molecular orbital (HOMO) and lowest unoccupied molecular orbital (LUMO). FMO theory has successfully been applied to elucidate the selectivity of aromatic substitution and pericyclic reactions, among others. As an example, the observed preference for nitration at the 1-position on the naphthalene molecule can be easily understood by considering the HOMO coefficients.

FMO theory has also been extended to solid and surface systems. Hoffmann and co-workers ${ }^{8}$ used the concepts of FMO theory and of orbital interactions to intuitively explain the electronic properties of solid states and surface-adsorbate interactions. In previous work, they have discussed the adsorption of small molecules and molecular fragments, such as $\mathrm{CO}^{9,10}$ and $\mathrm{CH}_{3},{ }^{11,12}$ on metal surfaces within a tight binding scheme based on the extended Hückel method. ${ }^{13}$ The interactions between the frontier orbitals of these molecules and the $d$ band of the metal surface make a major contribution to the adsorption process as well as to the energies of transition states on surfaces. The center of the $d$ band is linearly related to the adsorption energies of atoms and molecules and to the energy barriers for various reactions, and therefore the $d$ band center has been used to identify and interpret trends in surface properties. ${ }^{14}$ 
Scaling relationships, which generally describe correlations between the binding energies of adsorbates, are powerful tools for the rational design of catalysts, since they allow the prediction of trends in reactivity and selectivity across material surfaces. ${ }^{15}$ Such correlations can dramatically reduce the otherwise large search space required to identify improved catalytic materials to a low-dimensional space of just one or two properties, known as descriptors, that are often simple to calculate using density functional theory (DFT) methods. In fact, DFT-based high-throughput screenings have successfully identified new catalysts for steam reforming, ${ }^{16}$ the hydrogen evolution reaction, ${ }^{17}$ the selective hydrogenation of acetylene, ${ }^{18}$ the oxygen reduction reaction, ${ }^{19}$ the ammonia decomposition reaction, ${ }^{20}$ ammonia synthesis, ${ }^{21}$ and other processes. As an example, Nørskov and co-workers discovered active $\mathrm{Cu}-\mathrm{Ni}$ and $\mathrm{Ni}-\mathrm{Ga}$ catalysts for the selective reduction of $\mathrm{CO}^{22}$ and $\mathrm{CO}_{2}{ }^{23}$ to methanol, respectively, guided by the volcano-shaped relationship between turnover frequency (TOF) and the oxygen atom adsorption energy.

Although scaling relationships for the purpose of analyzing solid catalysts have thus far been primarily applied to pure transition metal surfaces,${ }^{24}$ scaling behaviors have also been identified for other catalytic surfaces, such as those of transition metal oxides, sulfides, and nitrides. ${ }^{25,26}$ Fernández et al. ${ }^{25}$ showed that the adsorption energies of $\mathrm{AH}_{x}$ intermediates $(\mathrm{A}=\mathrm{O}, \mathrm{S}, \mathrm{N})$ scale with those of the bare $\mathrm{A}$ atoms on transition metal oxide, sulfide, and nitride surfaces. Their work also identified that the slopes of the linear correlations are independent of the metal and are only affected by the number 
of $\mathrm{H}$ atoms in the molecule. Adsorption energies are key predictors of catalysis on metal oxides, because adsorption is a fundamental step in surface-catalyzed reactions. In addition, the molecular adsorption energies typically correlate with the energies of reaction intermediates and transition states across a wide range of catalyst surfaces. Therefore, determining the adsorption energies of reactant molecules on solid surfaces enables a descriptor-based analysis of the reactivity and selectivity of various catalysts. Despite this, the development of scaling relationships for adsorption energies on transition metal oxide surfaces remains a challenge, ${ }^{27}$ due to the extreme variations ${ }^{28}$ in the structure and properties of such materials and to computational difficulties. ${ }^{29}$

Titanium oxide $\left(\mathrm{TiO}_{2}\right)$ is widely used in heterogeneous catalysis, ${ }^{30}$ photocatalysis, ${ }^{31}$ solar cells, ${ }^{32}$ and batteries ${ }^{33}$ because it is abundant, non-toxic and highly stable under a variety of conditions. The major structural forms of $\mathrm{TiO}_{2}$ are anatase, rutile, and brookite. Rutile is the most common naturally-occurring form and is widely used as a white pigment, while anatase is considered to be the most photocatalytically active polymorph. ${ }^{34,35}$ The structures and properties of these forms have been investigated in the field of surface science so as to improve the performance of $\mathrm{TiO}_{2}$ as a catalyst. DFT calculations have successfully predicted $\mathrm{TiO}_{2}$ properties and also served to explain experimental observations of $\mathrm{TiO}_{2}$ systems over the last 20 years. ${ }^{36}$ Surface energies are essential for understanding the phase stability and surface reactivity in catalytic systems but, because these energies are difficult to measure experimentally, they are often obtained from DFT calculations. The surface energies of 
relaxed $\mathrm{TiO}_{2}$ surfaces have been ranked as $(101)<(100)<(001)<(110)$ for anatase and $(110)<(100)<(101)<(001)$ for rutile..$^{37,38}$ These computational results are in good agreement with the observed predominance of the (101) and (110) surfaces in anatase and rutile, respectively.

The adsorption of small molecules such as $\mathrm{H}_{2} \mathrm{O},{ }^{39} \mathrm{O}_{2},{ }^{40}$ methanol, ${ }^{41}$ and formic acid ${ }^{42}$ on $\mathrm{TiO}_{2}$ surfaces has been studied extensively due to the application of such processes in catalysis, using both experimental and theoretical methods. ${ }^{43,44}$ In particular, the interaction of water with $\mathrm{TiO}_{2}$ surfaces has been the subject of many studies, with the aim of understanding the mechanism of photochemical water splitting on such surfaces. Water molecules have been found to adsorb on the top of unsaturated five-fold $\left(\mathrm{Ti}_{5 \mathrm{c}}\right)$ coordinated $\mathrm{Ti}$ atoms, in conjunction with the hydrogen-bonding of $\mathrm{H}$ atoms to two bridging $\mathrm{O}_{2 \mathrm{c}}$ atoms on the anatase (101) surface. This structure, first predicated theoretically, ${ }^{45}$ has been confirmed by various experimental techniques, including atomic-scale scanning tunneling microscopy images. ${ }^{46,47}$ The interaction of $\mathrm{O}_{2}$ with $\mathrm{TiO}_{2}$ surfaces is of direct importance in photocatalytic processes because $\mathrm{O}_{2}$ acts as an electron scavenger and is often used to suppress electron-hole recombination, resulting in an increase in the lifetime of excited states. ${ }^{48}$ Although experimental investigations regarding the adsorption of organic molecules on $\mathrm{TiO}_{2}$ surfaces have been relatively scarce, the adsorption and dissociation of methanol and formic acid have been extensively explored due to their potential to serve as energy carriers. ${ }^{49}$ 
Despite intensive efforts to understand molecular adsorption on $\mathrm{TiO}_{2}$ surfaces, the range of molecules investigated as adsorbates is still limited. In addition, these studies were conducted under many different conditions (including variations in temperature, pressure, heating rate, reaction time, and support material), which prevents a direct comparisons of key properties, such as adsorption/desorption energy, coverage, and adsorption mode. For these reasons, the present study focused on the analysis of molecular adsorption on $\mathrm{TiO}_{2}$ surfaces using DFT computations and state-of-the-art machine learning (ML) techniques. This work is meant to be a first step toward building a comprehensive understanding of molecular adsorption on metal oxide surfaces from a frontier orbital viewpoint. The adsorption of 41 small molecules on anatase (101) and rutile (110) surfaces was systematically investigated at a uniform, consistent level of theory to determine a set of descriptors that summarize the adsorption processes. The results show that the mechanistic, energetic, and structural aspects of such molecular adsorption, which at first glance might appear complicated, can be regarded simply as an outcome of FMO theory, as expected from the importance of this theory in defining chemical structures and reactivities.

\section{Computational method}


All calculations were performed using the projector augmented-wave method $^{50}$ as implemented in the VASP code. ${ }^{51,52}$ The PBEsol functional was selected from among the generalized gradient approximations (GGAs) because it provides reasonable approximations of crystal structures and their surfaces. ${ }^{53-55}$ Plane wave basis sets with a cutoff energy of $400 \mathrm{eV}$ were used for all calculations and the empirical Grimme D3 method ${ }^{56}$ with Becke-Johnson damping ${ }^{57}$ was employed to apply a van der Waals correction. The Brillouin zone was sampled with $(2 \times 2 \times 1)$ Monkhorst-Pack $^{58}$ k-points and the anatase $\mathrm{TiO}_{2}$ (101) surface was modeled using a supercell slab containing 108 atoms (36 $\mathrm{Ti}$ and $72 \mathrm{O}$ ). As shown in Figure S1, the slab comprised three $\mathrm{Ti}_{12} \mathrm{O}_{24}$ layers in the [101] direction, separated in the vertical direction by a vacuum space with a height of $15 \AA$. The bottom atomic layer was fixed at the corresponding bulk positions. The adsorption energies $\left(E_{\text {ads }}\right)$ of the adsorbates were defined as

$$
E_{\mathrm{ads}}=E_{\mathrm{M} / \mathrm{S}}-E_{\mathrm{M}}-E_{\mathrm{S}}
$$

where $\mathrm{M}$ represents an adsorbate and $\mathrm{S}$ represents the surface.

\section{Results and discussion}

\section{3-1. HOMO and LUMO levels of small molecules}

Determining the HOMO and LUMO levels of isolated molecules is challenging within the framework of periodic DFT computations ${ }^{59}$ because these levels must be ascertained relative to an appropriate reference state. In addition, interactions 
between molecules in neighboring unit cells can be non-negligible. Thus, we carefully evaluated the orbital levels using three approaches. Firstly, non-periodic DFT computations of isolated molecules were performed with the Gaussian 09 program, ${ }^{60}$ secondly, periodic DFT computations of essentially isolated molecules were carried out in conjunction with a large cubic unit cell, and lastly periodic DFT computations were performed, applying a slab model in which the target molecule is kept away from the $\mathrm{TiO}_{2}$ surface. Table $\mathrm{S} 1$ provides the computed HOMO and LUMO levels for the small molecules obtained using these methods. In the case of the non-periodic model, all geometries were fully optimized at the PBE/6-311G(d,p) level of theory. ${ }^{61}$ It is worth noting that the addition of diffuse functions decreased correlations between the orbital levels obtained with the three methods. In the isolated periodic model, the target molecules were placed in the middle of a large empty unit cell $(30 \times 30 \times 30 \AA)$ to mimic isolated species, after which the HOMO and LUMO levels were calculated from the vacuum level. In the slab model, one atom of the target molecule was fixed throughout the geometric optimization so as to hold the molecule at least $5 \AA$ from the $\mathrm{TiO}_{2}$ surface. The HOMO and LUMO levels were calculated from the valence band maximum (VBM) of the $\mathrm{TiO}_{2}$ surface. As shown in Figure 1, highly linear correlations are apparent between the orbital levels calculated using all three methods, especially in the case of the HOMO values. Judging from the excellent linear correlation between the isolated periodic and slab models, interactions between the molecules and surface are likely to be negligible. The slope of each plot is close to 1 , with the exception of the 
LUMO values obtained using the non-periodic model plotted against the values from the isolated periodic (1.42) and slab (1.30) models. Because the PBEsol functional is not implemented in the Gaussian program, we used the PBE functional for the non-periodic calculations, which could have produced the steeper slopes. However, overall, these results confirm that the HOMO and LUMO levels of the isolated molecules relative to the vacuum or VBM level were successfully determined using periodic DFT computations.
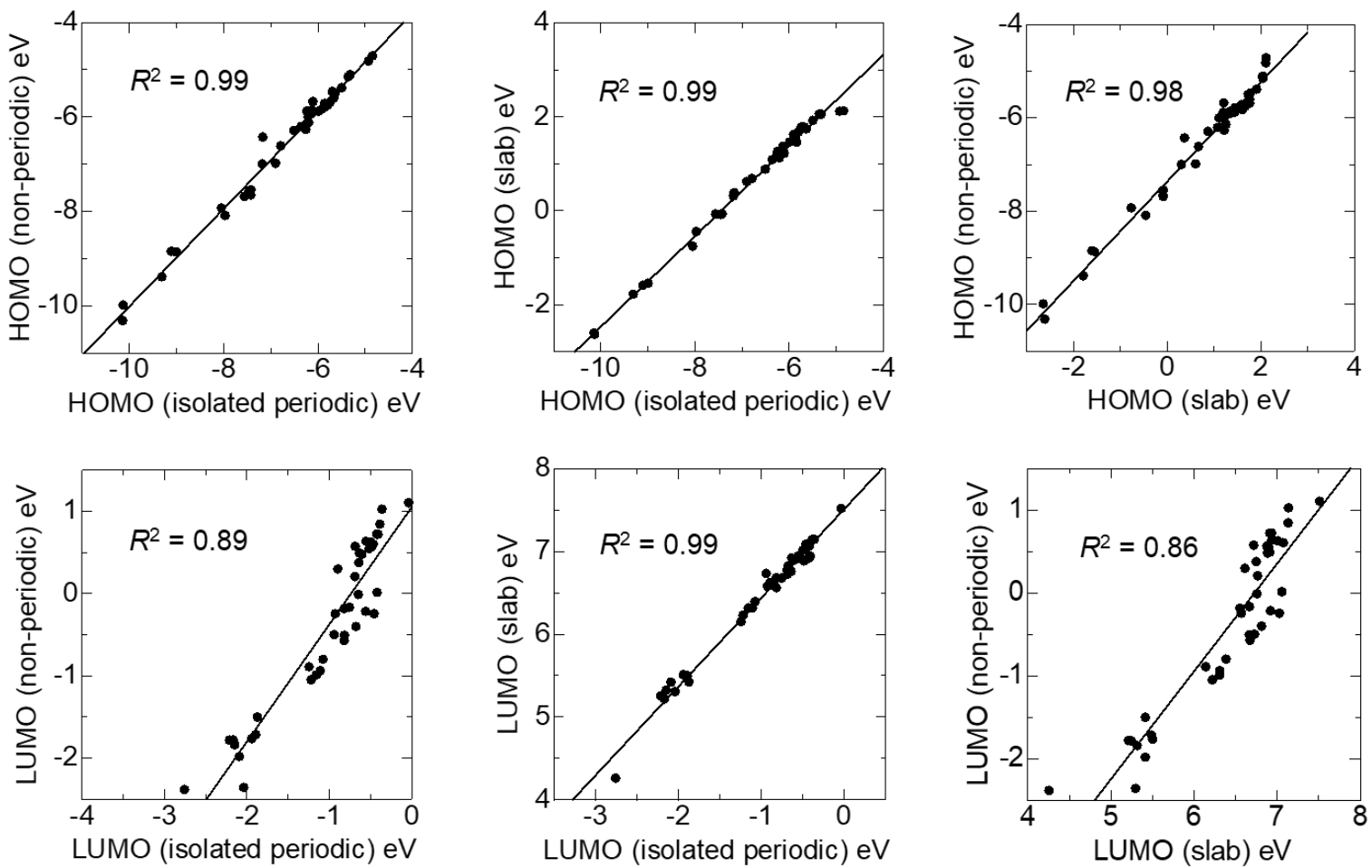

Figure 1. Correlations of values for the frontier orbital levels of small molecules obtained using the three different calculation methods.

The computed HOMO (LUMO) levels (Table S1) range from -10.13 (-2.75) $\mathrm{eV}$ to $-4.84(-0.03) \mathrm{eV}$ in the case of the isolated periodic model. The lowest HOMO levels were determined for $\mathrm{H}_{2}, \mathrm{~N}_{2}, \mathrm{CO}_{2}, \mathrm{CO}$, and acetonitrile because of their larger $s$ 
character and lower $p$ orbital levels associated with these compounds. $\mathrm{CH}_{4}$ has a threefold degenerate $\mathrm{HOMO}$ at a relatively low energy level of $-9.30 \mathrm{eV}$ because of the high degree of symmetry of the molecule. ${ }^{62}$ The HOMO levels of the saturated hydrocarbons are somewhat higher, and the HOMO levels of many of these molecules (including the unsaturated hydrocarbons, alcohols, and compounds containing a $\mathrm{C}=\mathrm{O}$ group) lie within the narrow range of -6.5 to $-5.5 \mathrm{eV}$. The HOMO levels of the amines are situated at the highest energy levels as a result of the lone pair orbitals on their nitrogen atoms, which are located at high energies and are also destabilized by the presence of electron-donating groups. As expected, the lowest LUMO levels were associated with the electrophilic molecules: formaldehyde, acetone, pyridine, $\mathrm{CHCl}_{3}$, and $\mathrm{CO}$.

Figure 2 presents a plot of LUMO levels versus HOMO levels for the small molecules, as determined using the isolated periodic model. There is no significant correlation in this plot, suggesting that the HOMO and LUMO levels are independent electronic properties of these molecules. This result also confirms that the HOMO and LUMO levels can be used independently as descriptors while developing ML models. 


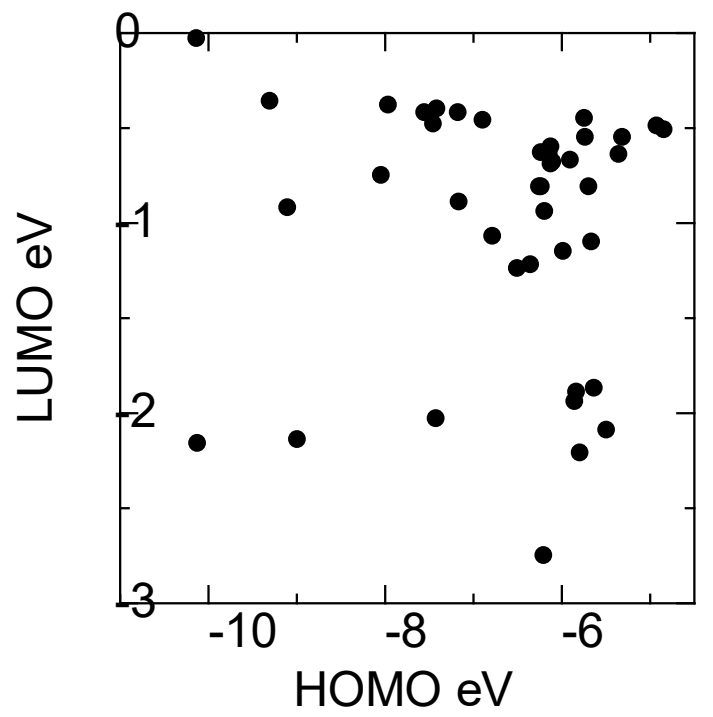

Figure 2. A plot of HOMO levels versus LUMO levels for the small molecules as calculated using the isolated periodic model.

\section{3-2. Molecular Adsorption on $\mathrm{TiO}_{2}$ surfaces}

\section{3-2-1. Anatase $\mathrm{TiO}_{2}(101)$ surface}

As discussed in the Introduction, $\mathrm{TiO}_{2}(101)$ is the most stable form of this oxide and also one of the most extensively studied anatase facets. For this reason, we initially investigated the adsorption of the small molecules on this surface at low coverages. A number of possible adsorption sites and molecular configurations were explored while searching for the most stable adsorption configurations, and the results are summarized in Figure S2 and Table S2. It was determined that the molecules are preferentially adsorbed on the tops of unsaturated five-fold coordinated $\mathrm{Ti}$ atoms. Various types of bonding between the molecules and the surface are included in the computed adsorption configurations, including lone pair-Ti bonds, $\pi$-electron donations and van der Waals interactions. The adsorption energies range from -0.2 to $-1.7 \mathrm{eV}$ and 
chemically hard molecules $\left(\mathrm{N}_{2}, \mathrm{CH}_{4}\right.$, and $\left.\mathrm{CO}\right)$ are weakly bound whereas amines and pyridine (having lone pairs on their nitrogen atoms) are strongly bound.

As shown in Figure 3, we plotted the computed adsorption energies of these molecules as functions of their HOMO and LUMO levels to ascertain any correlations between these variables. A linear relationship is evident between the adsorption energy and the HOMO level, with a coefficient of determination, $R^{2}$, of 0.64 . In contrast, there is no obvious correlation in the case of the LUMO level. Some degree of correlation between the adsorption energy and the HOMO level was expected because the most important factor associated with adsorption is the Lewis acid-base interaction between Ti cations (acting as Lewis acid sites) and the small molecules (acting as Lewis bases). In this process, the HOMOs of the molecules are stabilized by the unfilled band associated with the oxide surface, and the HOMOs become closer in energy to the unfilled band going from left to right in Figure 3a. Even though the correlation was not perfect, it is still impressive and suggests the possibility of predicting molecular adsorption outcomes at lower computational cost.

(a)

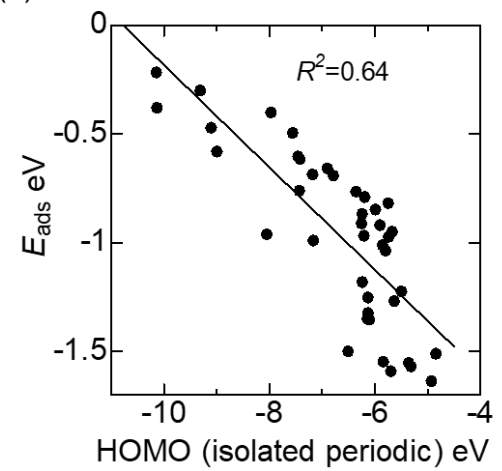

(b)

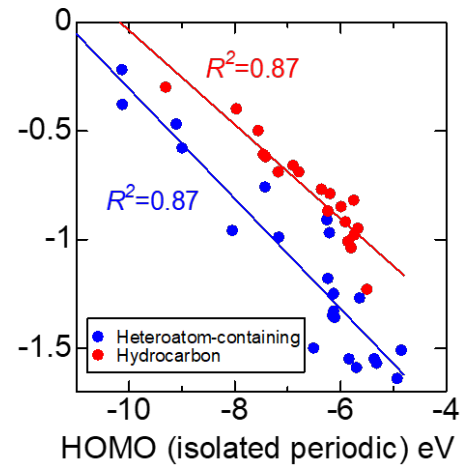

(c)

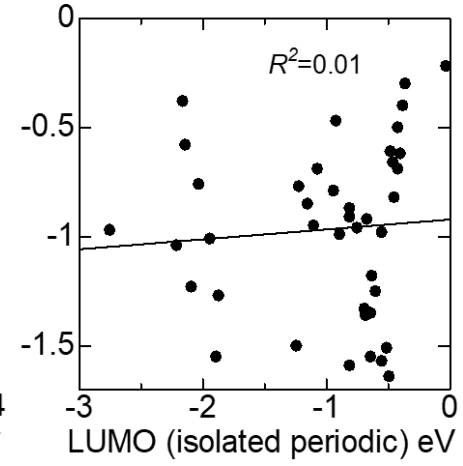

Figure 3. Adsorption energies $E_{\text {ads }}$ as functions of (a and b) HOMO and (c) LUMO levels for an anatase $\mathrm{TiO}_{2}(101)$ surface. 
Interestingly, a higher degree of correlation $\left(R^{2}=0.87\right)$ between the adsorption energy and the HOMO level can be obtained by dividing the small molecules into two subgroups: hydrocarbons and heteroatom-containing compounds (Figure 3b). This effect can likely be ascribed to the fundamentally different adsorption mechanisms of these two classes of compounds, which involve physisorption and chemisorption, respectively. Hydrocarbons are adsorbed primarily by van der Waals interactions, which are weak and typically non-directional. In contrast, heteroatom-containing compounds can form chemical bonds with surface atoms, such that significant orbital interactions take place between the molecules and surface. The residual scatter plot (residual versus predicted value) is shown in Figure S3. The residuals are split almost evenly between positive and negative. Hydrocarbons and heteroatom-containing compounds are located mainly in the positive and negative region, respectively, as expected. Large deviations from the linear relationship were found for the three amines, acetic acid, and pyridine. The adsorption of acetic acid and pyridine are aided by hydrogen bonds with the surface in addition to the chemical bonds. The amines have the highest HOMO levels among the adsorbates and the adsorption energy might not correctly predicted by the linear relationship. The slope of the linear relationship for the heteroatom-containing compounds is nearly identical to that for the hydrocarbons, although the intercept of the former plot is $0.63 \mathrm{eV}$ lower. The linear correlation between the adsorption energies of the hydrocarbons and the HOMO level is rather surprising. As noted, the adsorption of hydrocarbons is dominated by van der Waals interactions, which originate from the instantaneous interactions of electrons. Thus, FMO theory cannot, in principle, be 
applied to systems based on van der Waals forces. The linear relationship between the van der Waals interaction and HOMO levels was confirmed by estimating the energy contribution of these forces to the adsorption energy by performing single-point calculations and geometric optimization without the dispersion correction. In these calculations, we considered 10 hydrocarbons that covered the entire range of HOMO levels. As shown in Figure 4, the interactions between the hydrocarbons and the surface were found to be very weak because the GGA functionals do not provide the long-range $\left(r^{-6}\right)$ attractive dispersion term that arises from non-local electron-electron correlations. $^{63}$ This result provides evidence that the adsorption energies of hydrocarbons are primarily due to van der Waals interactions. However, based on this finding, it is difficult to explain why the adsorption energies show a linear correlation with the HOMO levels. Although there may be no direct link between the van der Waals interactions and the HOMO levels, Van der Waals forces do increase with molecular size, and the HOMO level is also correlated with size (Figure S4). ${ }^{64}$ Thus, the most likely interpretation is that the apparent correlation between the two variables is spurious, but could still be employed to estimate the adsorption energies for a wide variety of small molecules. It should be noted that the dipole moments of the molecules could also contribute to the adsorption process, which might help to explain the observed difference between hydrocarbons and heteroatom-containing compounds (see below). 


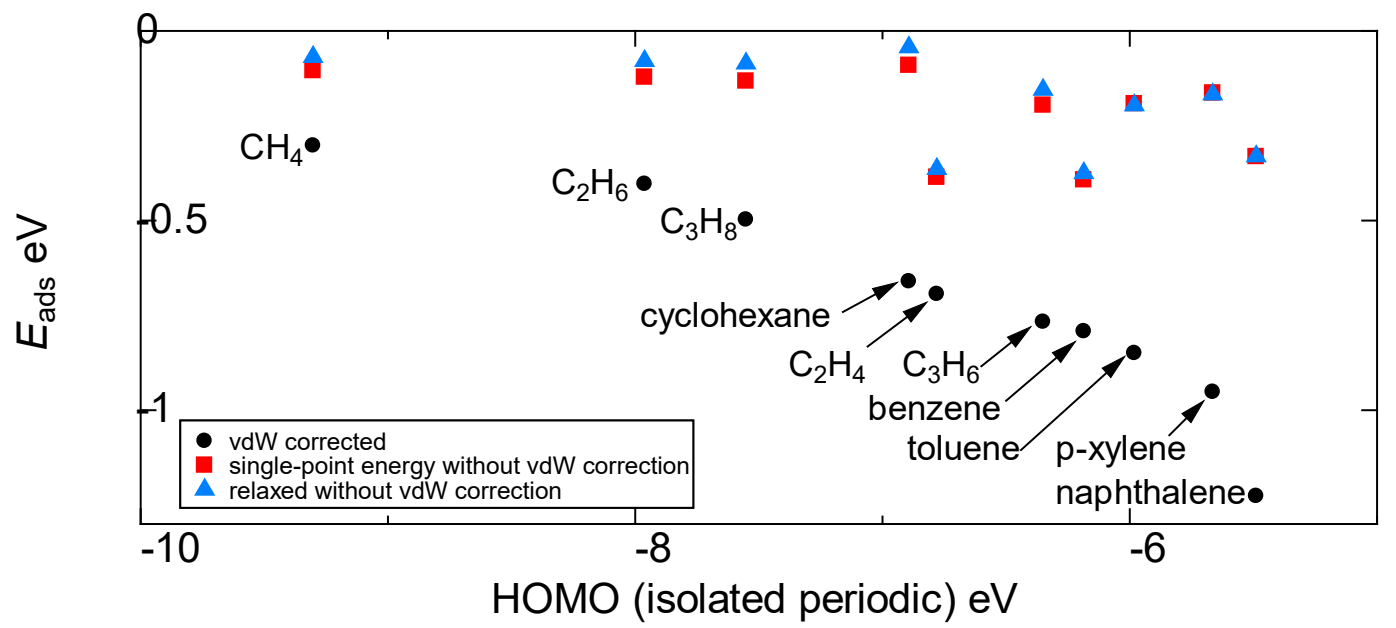

Figure 4. The effect of dispersion correction on the adsorption energies of hydrocarbons. Single-point calculations were performed using vdW-corrected structures.

\section{3-2-2. Rutile $\mathrm{TiO}_{2}(110)$ and less stable surfaces}

On the rutile $\mathrm{TiO}_{2}$ (110) surface, Ti atoms are situated in rectangular arrangements with six-fold coordinated $\mathrm{Ti}$ atoms at the corners and five-fold coordinated $\mathrm{Ti}$ atoms at the centers. Two types of oxygen atoms are also included: three-fold coordinated oxygen atoms located in the surface plane and two-fold coordinated bridging oxygen atoms, the latter of which protrude from the surface by approximately $1 \AA$ and form rows along the surface in the [001] direction. Based on the Wulff construction and computed surface energies, the most stable $\mathrm{TiO}_{2}(110)$ surface dominates the predicted equilibrium shape of a macroscopic crystal. ${ }^{65}$ Thus, we examined the adsorption of small molecules on a rutile $\mathrm{TiO}_{2}$ (110) surface. We considered the adsorption of 29 arbitrarily chosen molecules, as summarized in Figure S5 and Table S3. As discussed later, the obtained linear correlation between the adsorption energy and HOMO levels was essentially identical to that for the anatase 
(101) surface, and therefore we did not perform further calculations for the remaining 12 molecules. The data show that the molecules are preferentially adsorbed on the tops of unsaturated five-fold coordinated $\mathrm{Ti}$ atoms in the most stable adsorption configuration.

Figure 5 demonstrates that molecular adsorption on a rutile (110) surface shows a trend similar to that on the anatase (101) surface. A significant linear relationship is observed between the adsorption energies and the HOMO levels (Figures $5 \mathrm{a}$ and $\mathrm{b}$ ), whereas no obvious correlation is seen in the case of the LUMO levels (Figure 5c). The slope and intercept of the plot between the adsorption energies and the HOMO levels are also nearly identical for the two surfaces: a slope of $-0.24(-0.24)$ and an intercept of $-2.54(-2.66) \mathrm{eV}$ for the anatase (101) (rutile (110)) surface, which reflects the nearly identical adsorption energies of the molecules between the surfaces, with a root mean square deviation of $0.11 \mathrm{eV}$ and maximum absolute deviation of 0.31 $\mathrm{eV}$ for trimethylamine. The bulky trimethylamine molecule is destabilized by steric interactions with the bridging oxygen atoms on the rutile (110) surface, resulting in the relatively high absolute deviation. These results indicate that both surfaces behave similarly with regard to molecular adsorption, although adsorption tends to be slightly stronger on the rutile (110) surface, judging from the intercept values and the computed surface energies of 37 and $51 \mathrm{meV} / \AA^{2}$ for the anatase (101) and rutile (110) surfaces, respectively (see Table S4). 
(a)

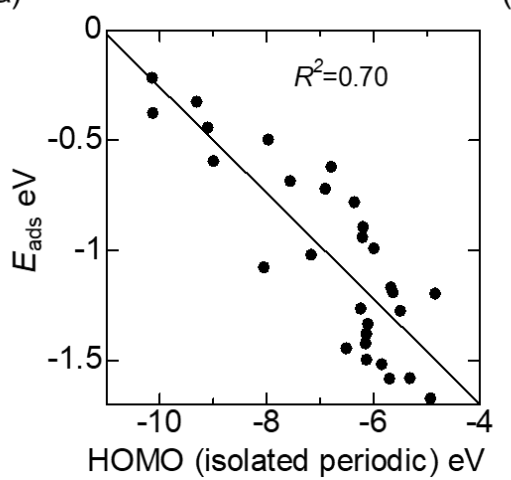

(b)

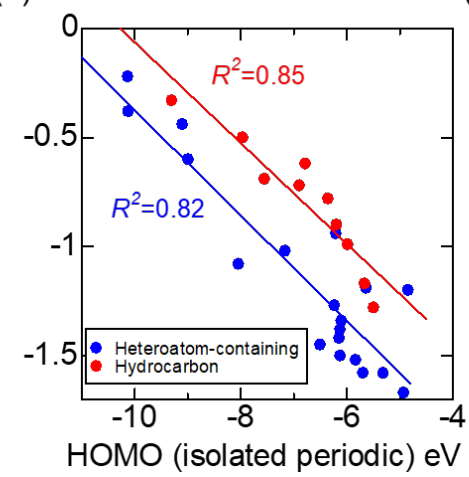

(c)

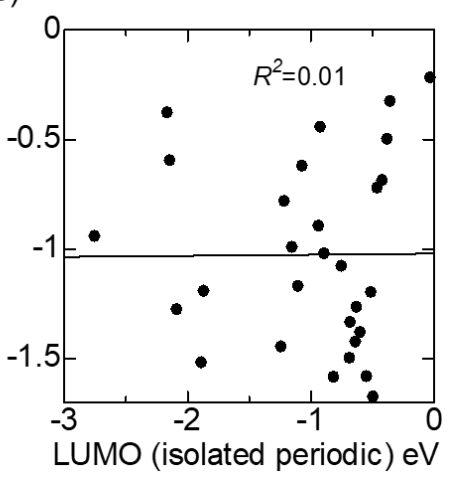

Figure 5. The adsorption energies $E_{\text {ads }}$ of small molecules as functions of the ( $\mathrm{a}$ and $\mathrm{b}$ ) HOMO and (c) LUMO levels for a rutile $\mathrm{TiO}_{2}(110)$ surface.

We examined the adsorption of acetone on seven unreconstructed $\mathrm{TiO}_{2}$ surfaces to identify good descriptors that exhibited a high degree of correlation with the adsorption energy. The carbonyl oxygen atom of acetone was found to coordinate to a Ti atom in the most stable adsorption configuration. The nucleophilic attack of a lattice oxygen on the carbonyl carbon also takes place on the anatase (001) and (110) and rutile (101) and (001) surfaces, resulting in the formations of $\mathrm{C}-\mathrm{O}$ and $\mathrm{Ti}-\mathrm{O}$ bonds. The adsorption energies were calculated to be $-1.27,-4.55,-1.77,-1.19,-1.61,-1.79$, and $-2.11 \mathrm{eV}$ for anatase (101), (001) and (110) and rutile (110), (100), (101) and (001) surfaces, respectively. We also observed a high correlation $\left(R^{2}=0.89\right)$ between the adsorption energies and surface energies and a moderate correlation $\left(R^{2}=0.32\right)$ between the adsorption energies and conduction band minimum (CBM) values, except for the anatase (001) surface, as shown in Figures 6 and S6. The acetone molecule is very strongly adsorbed on the anatase (001) surface, and this surface appears to be an outlier in the data set. On this surface, the $\mathrm{C}-\mathrm{O}$ bond is strongest because of its 
less-strained structure in the absence of a four-membered ring. The $\mathrm{C}-\mathrm{O}$ bond distances are $1.407,1.454,1.441$, and $1.441 \AA$ and the $\mathrm{O}-\mathrm{C}-\mathrm{O}$ bond angles are $111.0^{\circ}, 103.7^{\circ}$, $105.0^{\circ}$, and $102.5^{\circ}$ on the anatase (001) and (110) and rutile (101) and (001) surfaces, respectively. Thus, the adsorption energy decreases as the surface energy and CBM increase, but significant deviations from these basic trends can result from strong chemisorption on the surface.

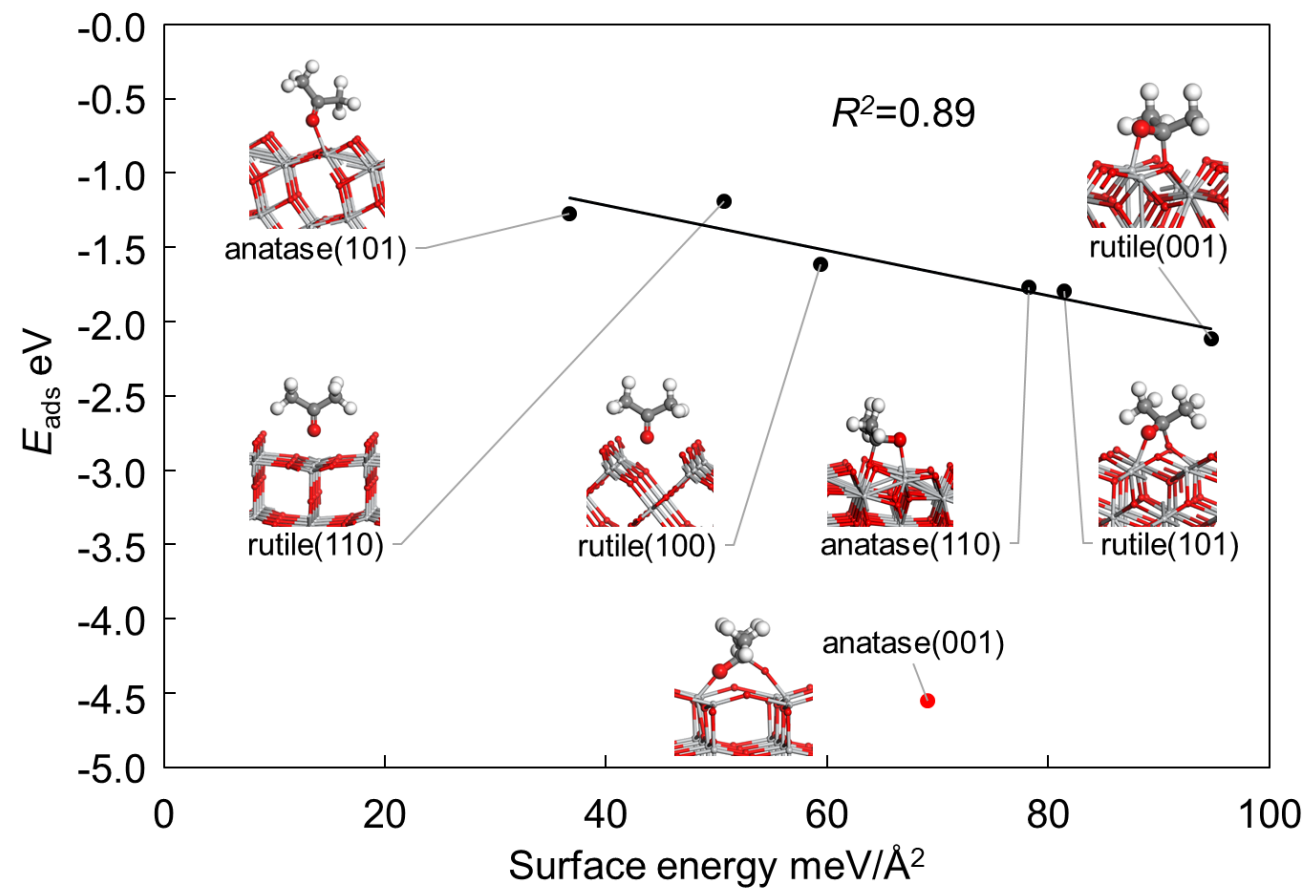

Figure 6. Adsorption energies $E_{\text {ads }}$ for acetone as a function of surface energies of seven $\mathrm{TiO}_{2}$ surfaces.

\section{3-3. Orbital Interactions between Small Molecules and Anatase $\mathrm{TiO}_{2}$ (101)}

\section{Surfaces}

In the previous sections, we found significant linear correlations between 
adsorption energies on $\mathrm{TiO}_{2}$ surfaces and the HOMO levels of small molecules, which suggest that the HOMO plays a central role in molecular adsorption. Here, we consider orbital interactions between the FMOs of molecules and the surfaces, so as to obtain a better understanding of the evident strong correlations. Figure 7 summarizes the density of states (DOS) for the anatase $\mathrm{TiO}_{2}(101)$ surfaces and the HOMO and LUMO levels of the isolated molecules. The bottom of the conduction band is slightly lower in energy than the HOMO levels of the amines, while the top of the valence band is close to the HOMO levels of propane and butane. As well, the HOMO levels of inert molecules, such as $\mathrm{N}_{2}$ and $\mathrm{CO}_{2}$, are somewhat lower than the VBM. As depicted in Figure 8, the HOMO and LUMO of each molecule mix with the surface states, resulting in the formation of a new HOMO and LUMO for the molecule absorbed on the surface. Very recently, Tsuji and Yoshizawa ${ }^{66}$ discussed orbital interactions between methane and rutile-type metal dioxides. Their work identified two-orbital two-electron interactions (termed simply A and B) based on charge-transfer. Interaction A corresponds to charge transfer from the HOMO of the molecule to an empty surface state, whereas interaction $\mathrm{B}$ is associated with a reverse charge-transfer process, in which electrons are donated to the LUMO of the molecule from a filled surface state. Two other interactions were also identified. Interaction $\mathrm{C}$ is a two-orbital four-electron process that leads to destabilization and repulsion between the molecule and surface, while interaction D generally does not contribute to molecule-surface bonding due to the lack of electron occupation. 


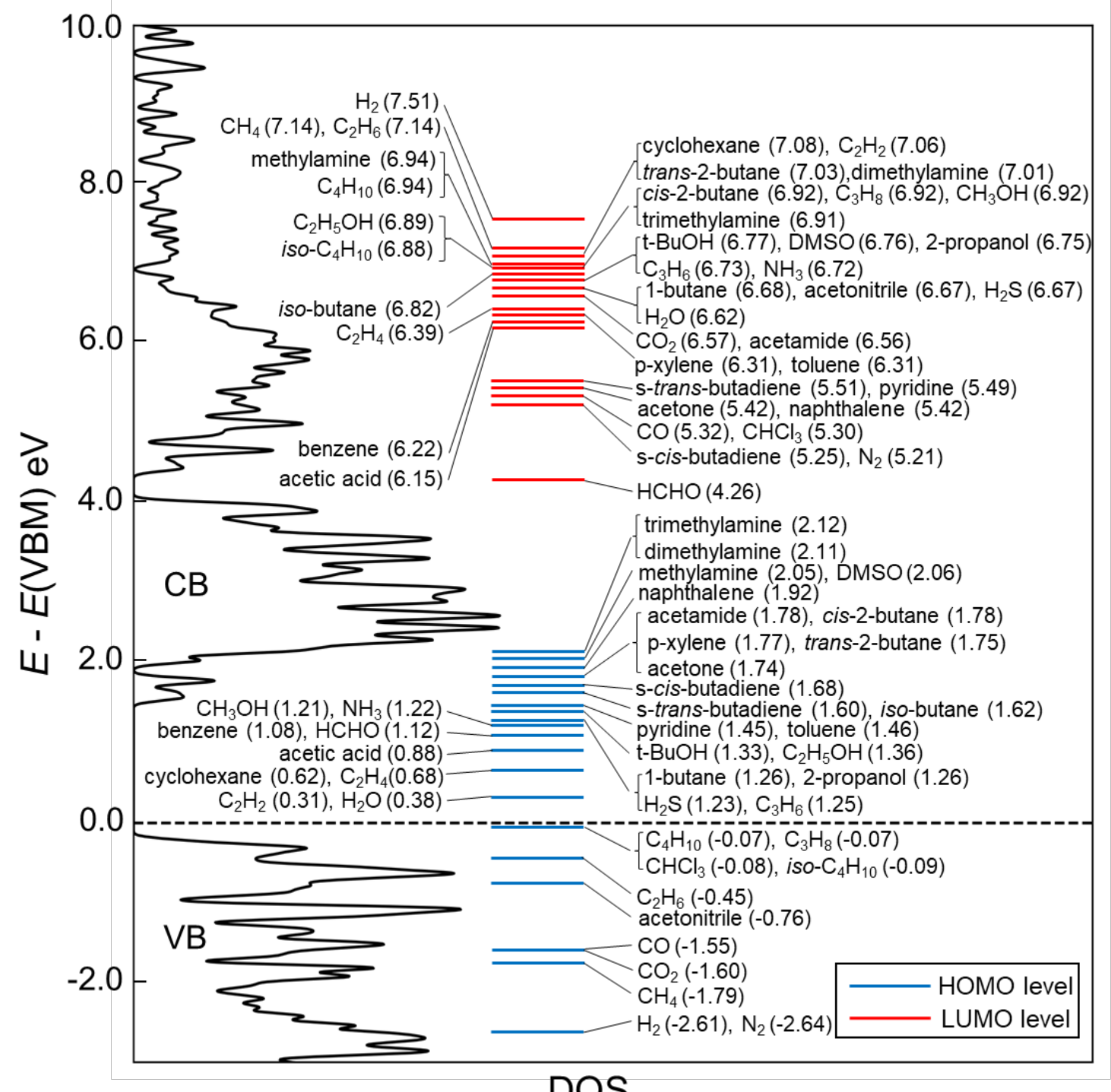

Figure 7. DOS data for an anatase $\mathrm{TiO}_{2}$ (101) surface and the HOMO and LUMO levels of isolated molecules. The HOMO and LUMO levels were determined relative to the VBM. 


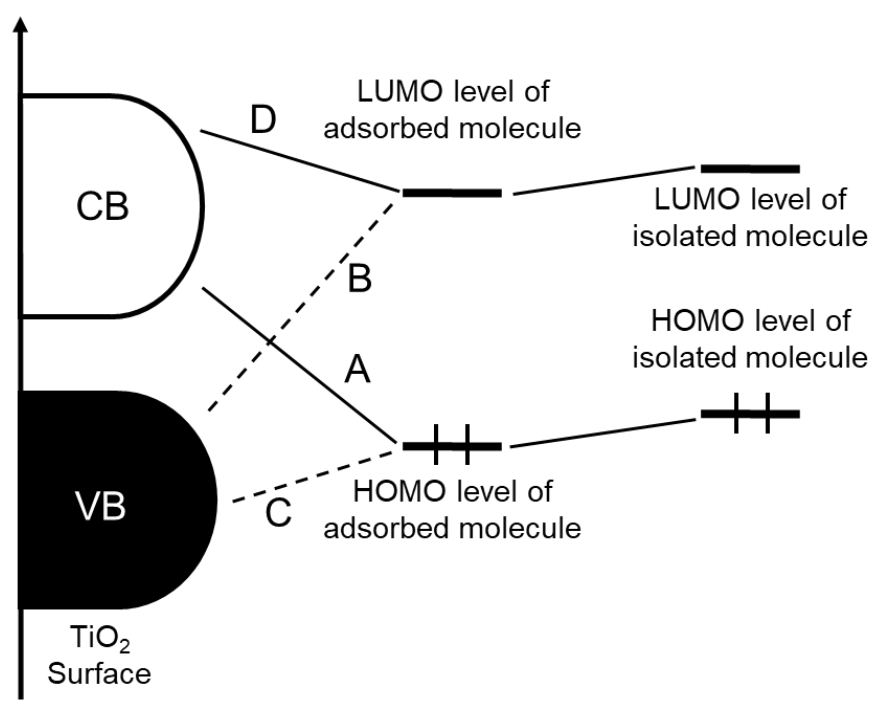

Figure 8. A schematic representation of the orbital interactions between the HOMO and LUMO of an isolated molecule and a $\mathrm{TiO}_{2}$ surface. Strong and weak orbital interactions are represented by solid and dashed lines, respectively.

We suggest that interactions $\mathrm{B}$ and $\mathrm{C}$ are not important for molecular adsorption on the $\mathrm{TiO}_{2}$ surface in contrast to the adsorptions on metal surfaces. Figure 9 shows the partial density of states (PDOS) plots for a vacant five-fold coordinated $\mathrm{Ti}$ site directly involved in molecular adsorption. The $d_{\mathrm{yz}}, d_{\mathrm{xz}}, d_{\mathrm{xy}}$, and $d_{\mathrm{z}^{2}}$ orbitals of Ti were found to be situated in the range from 2 to $4.3 \mathrm{eV}$ (the higher $d_{\mathrm{x}}^{2}-\mathrm{y}^{2}$ orbital is not shown). Although some Ti $d$ character mixes into the $p$ states of neighboring oxygen atoms, the d orbitals of Ti primarily form the surface conduction band. These results are consistent with previous studies, ${ }^{67,68}$ which established that the valence and conduction states are derived primarily from $\mathrm{O} 2 p$ and Ti $3 d$ orbitals, respectively. In the course of the molecular adsorption process, the molecule approaches a vacant five-fold coordinated $\mathrm{Ti}$ atom, although the extent of overlap between the FMO and the valence 
state remain close to zero due to the lack of $d$ character in the valence state. Based on perturbation theory, ${ }^{69}$ the energy change of the orbitals resulting from orbital interactions is given by

$$
\Delta \varepsilon_{i}=\frac{\left|H_{i j}{ }^{\prime}\right|^{2}}{\varepsilon_{i}^{(0)}-\varepsilon_{j}^{(0)}},
$$

where $\varepsilon_{i}^{(0)}$ is the unperturbed orbital energy prior to the interaction. The numerator of eq 1 is the square of the absolute value of the perturbation matrix element, which can be written as

$$
H_{i j}{ }^{\prime}=\left\langle\psi_{i}^{(0)}\left|H^{\prime}\right| \psi_{j}^{(0)}\right\rangle \text {. }
$$

Here, $\psi_{i}^{(0)}$ denotes an unperturbed wave function and $H^{\prime}$ represents the Hamiltonian operator for the perturbation. As discussed above, the matrix element between the HOMO and LUMO of the molecule and valence bands is likely to be negligible. Thus, the interactions $\mathrm{B}$ and $\mathrm{C}$ will be weak and the interaction between the HOMO and conduction state (interaction A) will be solely responsible for molecular adsorption. That is, charge transfer from the HOMO of the molecule to the Lewis acidic Ti site of the $\mathrm{TiO}_{2}$ surface is the dominant effect during molecular adsorption. These results might explain the high correlation of adsorption energy with the HOMO level and the lack of correlation with the LUMO level. However, further analysis is required to determine why the correlation between the adsorption energy and the HOMO level is almost linear. 


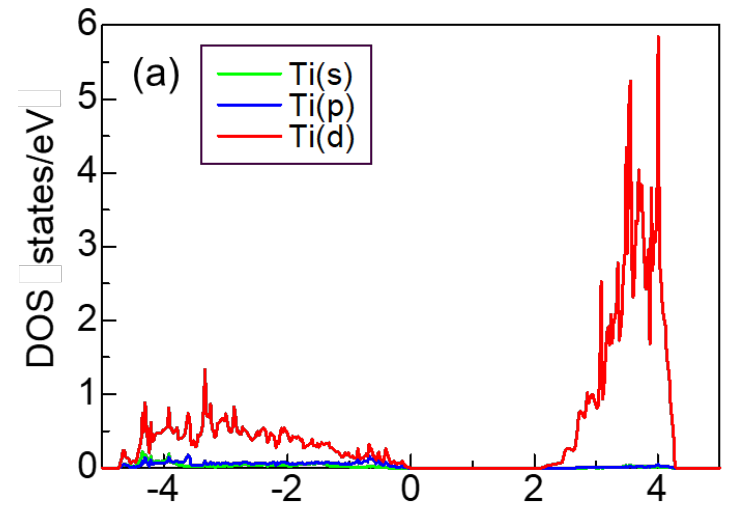

$E-E_{f} \mathrm{eV}$

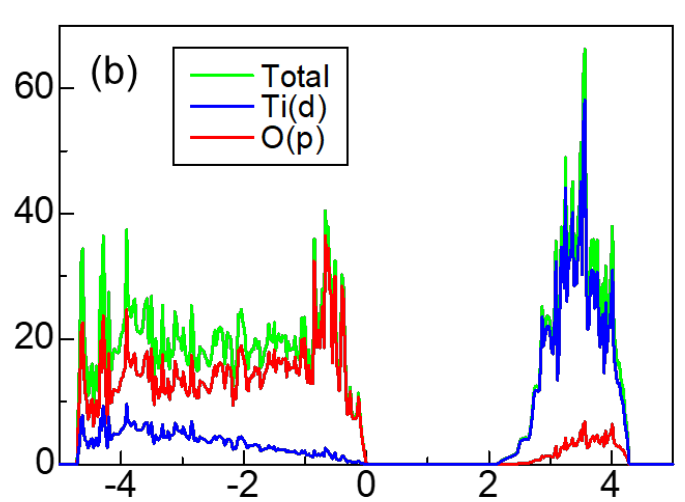

$E-E_{f} \mathrm{eV}$

Figure 9. Partial density of states (PDOS) plots for (a) a five-fold coordinated Ti atom and (b) surface atoms on an anatase (101) surface. The energy values were determined from the Fermi level $\left(E_{f}\right)$.

Finally, we generated a wide variety of descriptors (1342 in total) to identify other important parameters associated with molecular adsorption using the Dragon program. ${ }^{70}$ These descriptors ranged from very simple (such as the molecular weight and number of atoms) to highly complex mathematical transformations of the molecular structural information. These descriptors were primarily used to establish quantitative structure-property and structure-activity relationships (QSPR and QSAR), such that ML algorithms could be employed to fit models and thus to predict observable properties, such as IC50. The HOMO-1 level has been previously used as a quantum-chemical descriptor for reaction modeling. ${ }^{71-73}$ Thus, we calculated the HOMO-1 levels of the isolated molecules and the HOMO and HOMO-1 levels of the absorbed molecules, with the results provided in Table S5. The charge densities of all bands were also computed to identify the key orbitals of the absorbed molecules (see Figure S7). Because some molecular orbitals are considerably broadened upon adsorption, states associated with 
the top of the broad peak were selected as representative of the orbitals. As an example, the $\mathrm{CO} 5 \sigma$ orbital (that is, the HOMO) and $1 \pi$ orbital (that is, the degenerate HOMO-1) were found to be situated at -4.14 and $-5.36 \mathrm{eV}$, respectively, relative to the VBM (see Figure 10). The HOMO and HOMO-1 levels are 2.59 and $1.20 \mathrm{eV}$ lower relative to those of free $\mathrm{CO}(-1.55$ and $-4.34 \mathrm{eV})$, respectively, by the surface. In addition, the $\mathrm{HOMO}$ electron density isosurfaces demonstrate significant charge transfer from $\mathrm{CO}$ to a Ti atom, further lowering the HOMO level.

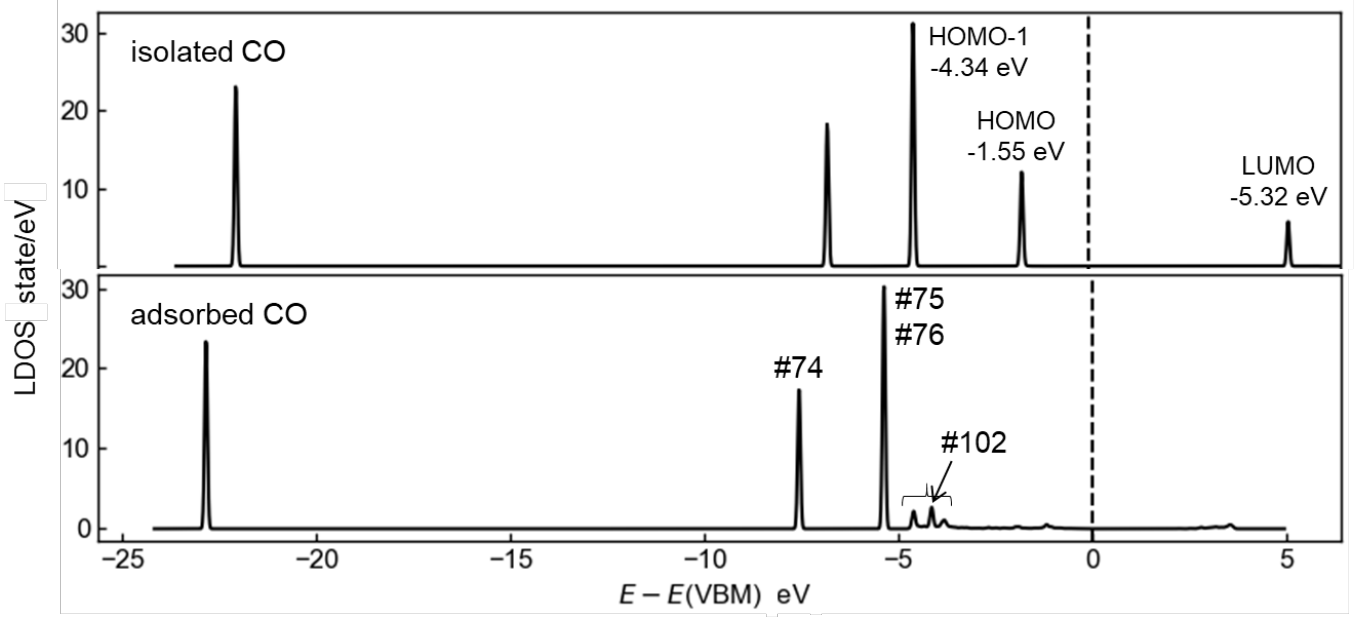

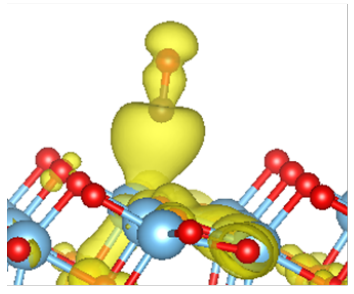

\#102 -4.14 eV

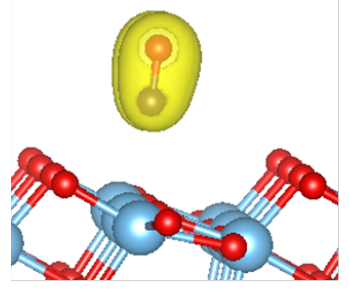

\#75, $76-5.36$ eV

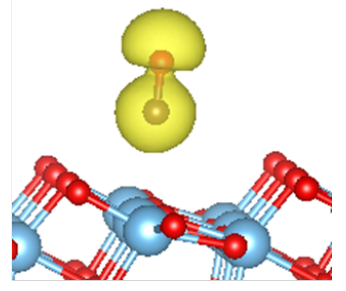

\#74 -7.56 eV

Figure 10. Density of states (DOS) data projected to the CO molecule and electron density isosurfaces for key orbitals. Energies were determined from the VBM of an anatase $\mathrm{TiO}_{2}$ surface. 
The descriptor correlation matrix was investigated to obtain a more complete view of the correlation between the orbital levels, as shown in Figure 11. As expected, the HOMO level of each isolated molecule was strongly correlated with the HOMO level of the absorbed molecule. Interestingly, the HOMO level was also moderately correlated with the HOMO-1 level, and hence the HOMO-1 data are statistically redundant and are not generally taken into consideration in FMO theory. We removed any descriptors that are correlated to each other by more than 0.25 to reduce overfitting and to obtain a simple interpretative model. 18 selected descriptors were listed in Table S6. The ExtraTrees algorithm ${ }^{74}$ was employed to rank the relative importance of the descriptors for the adsorption energy, and Figure 12a shows the 10 most important descriptors for the prediction of adsorption energy based on nonlinear regression. We also conducted variable importance (VIP) analysis ${ }^{75}$ to confirm that the results are similar (Figure S8). It is evident that the HOMO level of the isolated molecule is the most important descriptor and that the number of nitrogen and oxygen atoms $(\mathrm{nN}$ and nO) are also important. As shown in Figure 3b, two lines are required to represent the adsorption energies of hydrocarbons and heteroatom-containing compounds, which would lead to the relatively high feature importance scores of $\mathrm{nN}$ and $\mathrm{nO}$. 


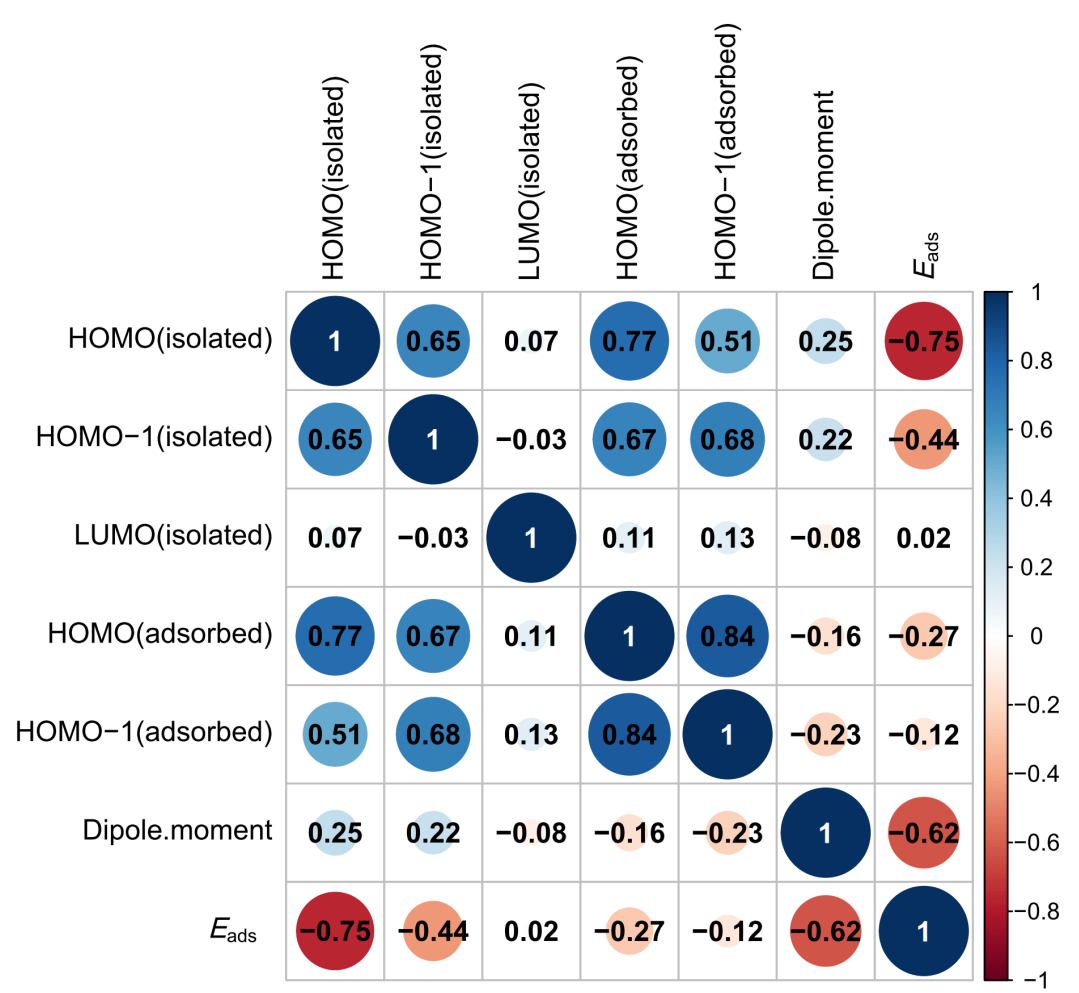

Figure 11. Correlation map for the orbital levels, dipole moments and adsorption energies.


Figure 12. Importance scores obtained using the ExtraTrees algorithm with 100 decision trees. (a) The orbital levels and Dragon-derived properties used as the descriptors and (b) after the dipole moment was added to the set of descriptors. 
Based on the above, we turned our focus to molecular properties as determined using DFT calculations, so as to improve our predictive capabilities, because the inclusion of DFT-based descriptors to a dataset often provides more accurate predictions. ${ }^{76}$ The dipole moments of small molecules in Table S7 were found to correlate with the adsorption energies but also showed a weak correlation with the HOMO levels (see Figure 11), which suggests the statistical significance of this parameter. As shown in Figure 12b, the HOMO level and dipole moment play the first and second most important roles in predicting the adsorption energy, respectively. Dipole interactions between the molecules and surface might contribute to the molecular adsorption process in conjunction with the orbital interactions between the HOMO and conduction band.

The predictive capability of the ML models was tested by dividing the data into training and test data sets, using a randomly selected third of the data as the test set. Figure 13 illustrates the predictive performance of the multiple linear regression (MLR) and ExtraTrees models with only HOMO levels and dipole moments as descriptors. In these plots, deviations from the $X=Y$ lines indicate errors in prediction. The root mean square error (RMSE) values for the differences between the predicted and DFT values for the test data were found to be 0.166 and $0.121 \mathrm{eV}$ for the MLR and ExtraTrees models, respectively. This value is slightly reduced, from 0.234 to $0.166 \mathrm{eV}$, by including the dipole moment in the descriptors for the linear model, which suggests that the dipole moment has a secondary effect on the adsorption of molecules on the $\mathrm{TiO}_{2}$ surface. Judging from the RMSE values, the nonlinear ExtraTrees model is slightly 
more accurate than the MLR model. However, it should be noted that linear models are generally more stable than nonlinear models while also being less flexible. Thus, stable linear models would be more appropriate for use during computational studies in the search for new heterogeneous catalysts, since reaction energetics in the current state-of-the-art computational screening ${ }^{22,23}$ have been treated in a drastically simplified manner based on a linearity assumption, such as the $d$-band model. ${ }^{27}$

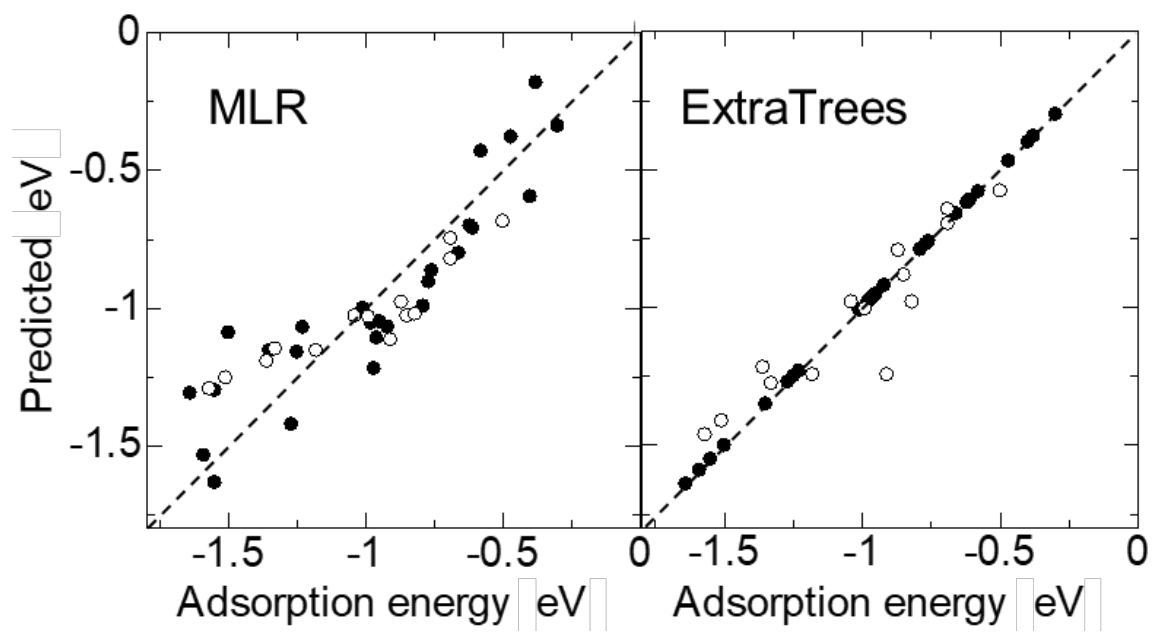

Figure 13. Adsorption energies of small molecules on an anatase $\mathrm{TiO}_{2}(101)$ surface and the predicted values obtained using the MLR and ExtraTrees methods. Training and test data are indicated by closed and open circles, respectively.

\section{Conclusions}

This work examined the adsorption of small molecules on $\mathrm{TiO}_{2}$ surfaces using periodic DFT calculations and statistical methods. The adsorption energies of 41 and 29 small molecules on anatase (101) and rutile (110) surfaces, respectively, were evaluated using the same computational method to obtain a uniform and consistent data set. We 
found linear relationships between the adsorption energies and the HOMO levels of molecules, whereas no obvious correlation was evident with regard to the LUMO levels. The adsorption energies of acetone on seven $\mathrm{TiO}_{2}$ surfaces were correlated with the surface energies, although strong chemisorption to the surface led to significant deviations from the basic trend. We analyzed the high degree of correlation between the HOMO levels and adsorption energies from the point of view of orbital interactions. Interactions between HOMOs and surface conduction states likely play a key role in molecular adsorption on $\mathrm{TiO}_{2}$ surfaces, and so the HOMO level shows the highest correlation among the descriptors explored in this study. ML-based statistical investigations indicate that the dipole moments of molecules also contribute to molecular adsorption. We believe that predictive, readily interpreted linear models such as those presented herein will serve as a guide to the future study of molecular adsorption on $\mathrm{TiO}_{2}$ surfaces at significantly reduced computational costs.

\section{Acknowledgments}

This study was funded by the Materials Research by Information Integration Initiative (MI2I) project of the Support Program for Starting Up Innovation Hubs through a grant (no. JPMJCR17J3), by the Innovative Catalysts program established by CREST, Japan Science and Technology Agency (JST), by a KAKENHI Grant-in-Aid (no. 18H01969) from the Japan Society for the Promotion of Science (JSPS), and by the Elements Strategy Initiative to Form Core Research Centers. Computing resources were 
provided by the Research Institute for Information Technology, Kyushu University and by the SuperComputer System, Institute for Chemical Research, Kyoto University.

Supporting Information Available One figure showing the slab model, two figures showing the adsorption structures, one figure showing the residual analysis of adsorption energy data, one figure showing the correlation between HOMO levels and number of carbon atoms, one figure showing the adsorption energies for acetone, one figure showing electron density isosurfaces, one figure showing the VIP analysis, six tables of numerical values from the statistical analysis, and one table of the computed surface energies for anatase and rutile $\mathrm{TiO}_{2}$. This material is available free of charge via the Internet at http://pubs.acs.org. 


\section{References}

(1) Fukui, K.; Yonezawa, T.; Shingu, H. A Molecular Orbital Theory of Reactivity in Aromatic Hydrocarbons. J. Chem. Phys. 1952, 20, 722-725.

(2) Fukui, K.; Yonezawa, T.; Nagata, C.; Shingu, H. Molecular Orbital Theory of Orientation in Aromatic, Heteroaromatic, and Other Conjugated Molecules. J. Chem. Phys. 1954, 22, 1433-1442.

(3) Fukui, K. Recognition of Stereochemical Paths by Orbital Interaction. Acc. Chem. Res., 1971, 4, 57-64.

(4) Woodward, R. B.; Hoffmann, R. Stereochemistry of Electrocyclic Reactions. J. Am. Chem. Soc., 1965, 87, 395-397.

(5) Hoffmann, R.; Woodward, R. B. Selection Rules for Concerted Cycloaddition Reactions. J. Am. Chem. Soc., 1965, 87, 2046-2048.

(6) Woodward, R. B.; Hoffmann, R. Selection Rules for Sigmatropic Reactions. J. Am. Chem. Soc., 1965, 87, 2511-2513.

(7) Hoffmann, R.; Woodward, R. B. Orbital Symmetries and endo-exo Relationships in Concerted Cycloaddition Reactions. J. Am. Chem. Soc., 1965, 87, 4388-4389.

(8) Hoffmann, R. How Chemistry and Physics Meet in the Solid State. Angew. Chem. Int. Ed., 1987, 26, 846-878.

(9) Glassey, W. V.; Hoffmann, R. A Comparative Study of the $\mathrm{p}(2 \times 2)-\mathrm{CO} / \mathrm{M}(111)$, M=Pt,Cu,Al Chemisorption Systems. J. Phys. Chem. B, 2001, 105, 3245-3260. 
(10) Glassey, W. V.; Hoffmann, R. A Molecular Orbital Study of Surface-Adsorbate Interactions during the Oxidation of CO on the Pt(111) Surface. Surf. Sci., 2001, 475, 47-60.

(11) Zheng, C.; Apeloig, Y.; Hoffmann, R. Bonding and Coupling of $\mathrm{C}_{1}$ Fragments on Metal Surfaces. J. Am. Chem. Soc., 1988, 110, 749-774.

(12) Papoian, G.; Nørskov, J. K.; Hoffmann, R. A Comparative Theoretical Study of the Hydrogen, Methyl, and Ethyl Chemisorption on the Pt(111) Surface. J. Am. Chem. Soc. 2000, $122,4129-4144$.

(13) Hoffmann, R. An Extended Hückel Theory. I. Hydrocarbons. J. Chem. Phys. 1963, $39,1397-1412$.

(14) Hammer, B.; Nørskov, J. K. Theoretical Surface Science and Catalysis Calculations and Concepts. Adv. Catal. 2000, 45, 71-129.

(15) Greeley, J. Theoretical Heterogeneous Catalysis: Scaling Relationships and Computational Catalyst Design. Annu. Rev. Chem. Biomol. Eng. 2016, 7, 605-635.

(16) Besenbacher, F.; Chorkendorff, I.; Clausen, B. S.; Hammer, B.; Molenbroek, A. M.; Nørskov, J. K.; Stensgaard I. Design of a Surface Alloy Catalyst for Steam Reforming. Science 1998, 279, 1913-1915.

(17) Greeley, J.; Jaramillo, T. F.; Bonde, J.; Chorkendorff, I.; Nørskov, J. K. Computational High-Throughput Screening of Electrocatalytic Materials for Hydrogen Evolution. Nat. Mater. 2006, 5, 909-913. 
(18) Studt, F.; Abild-Pedersen, F.; Bligaard, T.; Sørensen, R. Z.; Christensen, C. H.; Nørskov, J. K. Identification of Non-Precious Metal Alloy Catalysts for Selective Hydrogenation of Acetylene. Science 2008, 320, 1320-1322.

(19) Greeley, J.; Stephens, I. E. L.; Bondarenko, A. S.; Johansson, T. P.; Hansen, H. A.; Jaramillo, T. F.; Rossmeisl, J.; Chorkendorff, I.; Nørskov, J. K. Alloys of Platinum and Early Transition Metals as Oxygen Reduction Electrocatalysts. Nat. Chem. 2009, 1, $552-556$.

(20) Hansgen, D. A.; Vlachos, D. G.; Chen, J. G. Using First Principles to Predict Bimetallic Catalysts for the Ammonia Decomposition Reaction. Nat. Chem. 2010, 2, 484-489.

(21) Jacobsen, C. J. H.; Dahl, S.; Clausen, B. S.; Bahn, S.; Logadottir, A.; Nørskov, J. K. Catalyst Design by Interpolation in the Periodic Table: Bimetallic Ammonia Synthesis Catalysts. J. Am. Chem. Soc. 2001, 123, 8404-8405.

(22) Studt, F.; Abild-Pedersen, F.; Wu, Q.; Jensen, A. D.; Temel, B.; Grunwaldt, J.-D.; Nørskov, J. K. CO Hydrogenation to Methanol on $\mathrm{Cu}-\mathrm{Ni}$ Catalysts: Theory and Experiment. J. Catal. 2012, 293, 51-60.

(23) Studt, F.; Sharafutdinov, I.; Abild-Pedersen, F.; Elkjær, C. F.; Hummelshøj, J. S.; Dahl, S.; Chorkendorff, I.; Nørskov, J. K. Discovery of a Ni-Ga Catalyst for Carbon Dioxide Reduction to Methanol. Nat. Chem. 2014, 6, 320-324. 
(24) Ras, E.-J.; Louwerse, M. J.; Mittelmeijer-Hazeleger, M. C.; Rothenberg, G. Predicting Adsorption on Metals: Simple yet Effective Descriptors for Surface Catalysis. Phys. Chem. Chem. Phys. 2013, 15, 4436-4443.

(25) Fernández, E. M.; Moses, P. G.; Toftelund, A.; Hansen, H. A.; Martínez, J. I.; Abild-Pedersen, F.; Kleis, J.; Hinnemann, B.; Rossmeisl, J.; Bligaard, T. et al. Scaling Relationships for Adsorption Energies on Transition Metal Oxide, Sulfide, and Nitride Surfaces. Angew. Chem. Int. Ed. 2008, 47, 4683-4686.

(26) Man, I. C.; Su, H.-Y.; Calle-Vallejo, F.; Hansen, H. A.; Martínez, J. I.; Inoglu, N. G.; Kitchin, J.; Jaramillo, T. F.; Nørskov, J. K.; Rossmeisl, J. Universality in Oxygen Evolution Electrocatalysis on Oxide Surfaces. ChemCatChem 2011, 3, 1159-1165.

(27) Norskov, J. K.; Abild-Pedersen, F.; Studt, F.; Bligaard, T. Density Functional Theory in Surface Chemistry and Catalysis. Proc. Natl. Acad. Sci. 2011, 108, 937-943.

(28) Cox, P. The Surface Science of Metal Oxides; Cambridge University Press, 1996; pp 1-7, 158-200, and 264-266.

(29) Pacchioni, G. Modeling Doped and Defective Oxides in Catalysis with Density Functional Theory Methods: Room for Improvements. J. Chem. Phys. 2008, 128, 182505.

(30) Vohs, J. M. Site Requirements for the Adsorption and Reaction of Oxygenates on Metal Oxides Surfaces. Chem. Rev. 2013, 113, 4136-4163. 
(31) Schneider, J.; Matsuoka, M.; Takeuchi, M.; Zhang, J.; Horiuchi, Yu. Anpo, M.; Bahnemann, D. W. Understanding $\mathrm{TiO}_{2}$ Photocatalysis: Mechanisms and Materials. Chem. Rev. 2014, 114, 9919-9986.

(32) Hagfeldt A.; Boschloo G.; Sun L.; Kloo, L. Pettersson H.; Dye-Sensitized Solar Cells. Chem. Rev. 2010, 110, 6595-6663.

(33) Dylla A. G.; Henkelman, G.; Stevenson, K. J. Lithium Insertion in Nanostructured $\mathrm{TiO}_{2}$ (B) Architectures. Acc. Chem. Res. 2013, 46, 1104-1112

(34) Diebold, U The Surface Science of Titanium Dioxide. Surf. Sci. Rep. 2003, 48, 53-229.

(35) Hashimoto, K.; Irie, H.; Fujishima, A. $\mathrm{TiO}_{2}$ Photocatalysis: A Historical Overview and Future Prospects. Jpn. J. Appl. Phys. 2005, 44, 8269-8285.

(36) Angelis, F. D.; Valentin, C. D.; Fantacci, S.; Vittadini, A.; Selloni, A. Theoretical Studies on Anatase and Less Common $\mathrm{TiO}_{2}$ Phases: Bulk, Surfaces, and Nanomaterials. Chem. Rev. 2014, 114, 9708-9753.

(37) Vittadini, A.; Casarin, M.; Selloni, A. Chemistry of and on $\mathrm{TiO}_{2}$-Anatase Surfaces by DFT Calculations: A Partial Review. Theor. Chem. Acc. 2007, 117, 663-671.

(38) Perron, H.; Domain, C.; Roques, J.; Drot, R.; Simoni, E.; Catalette, H. Optimisation of Accurate Rutile $\mathrm{TiO}_{2}$ (110), (100), (101), and (001) Surface Models from Periodic DFT Calculations. Theor. Chem. Acc. 2007, 117, 565-574.

(39) Sun, C.; Liu, L.-M.; Selloni, A.; Lu, G. Q.; Smith, S. C. Titania-Water Interactions: A Review of Theoretical Studies. J. Mater. Chem. 2010, 20, 10319-10334. 
(40) Mattioli, G.; Filippone, F.; Bonapasta, A. A. Reaction Intermediates in the Photoreduction of Oxygen Molecules at the (101) $\mathrm{TiO}_{2}$ (Anatase) Surface J. Am. Chem. Soc. 2006, 128, 13772-13780.

(41) Gong, X.-Q.; Selloni, A. Role of Steps in the Reactivity of the Anatase $\mathrm{TiO}_{2}(101)$ Surface. J. Catal. 2007, 249, 134-139.

(42) Kou, L.; Frauenheim, Th.; Rosa, A. L.; Lima, E. N. Hybrid Density Functional Calculations of Formic Acid on Anatase $\mathrm{TiO}_{2}(101)$ Surfaces. J. Phys. Chem. C 2017, $121,17417-17420$.

(43) Pang, C. L.; Lindsay, R.; Thornton, G. Chemical Reactions on Rutile $\mathrm{TiO}_{2}(110)$. Chem. Soc. Rev. 2008, 37, 2328-2353.

(44) Thomas, A. G.; Syres, K. L. Adsorption of Organic Molecules on Rutile $\mathrm{TiO}_{2}$ and Anatase $\mathrm{TiO}_{2}$ Single Crystal Surfaces. Chem. Soc. Rev. 2012, 41, 4207-4217.

(45) Vittadini, A.; Selloni, A.; Rotzinger, F. P.; Grätzel, M. Structure and Energetics of Water Adsorbed at $\mathrm{TiO}_{2}$ Anatase (101) and (001) Surfaces. Phys. Rev. Lett. 1998, 81, 2954-2957.

(46) Herman, G. S.; Dohnálek, Z.; Ruzycki, N.; Diebold, U. Experimental Investigation of the Interaction of Water and Methanol with Anatase-TiO $2(101)$. J. Phys. Chem. B 2003, 107, 2788-2795.

(47) He, Y.; Tilocca, A.; Dulub, O.; Selloni, A.; Diebold, U. Local Ordering and Electronic Signatures of Submonolayer Water on Anatase $\mathrm{TiO}_{2}(101)$. Nat. Mater. 2009, $8,585-589$. 
(48) Li, Y.-F.; Aschauer, U.; Chen, J.; Selloni, A. Adsorption and Reactions of $\mathrm{O}_{2}$ on Anatase $\mathrm{TiO}_{2}$. Acc. Chem. Res. 2014, 47, 3361-3368.

(49) Zhang, Q.; Lin, C.-F.; Jing, Y. H.; Chang, C.-T. Photocatalytic Reduction of Carbon Dioxide to Methanol and Formic Acid by Graphene-TiO2. J. Air Waste Manag. Assoc. 2014, 64, 578-585.

(50) Blöchl, P. E., Projector Augmented-Wave Method. Phys. Rev. B 1994, 50 (24), 17953-17979.

(51) Kresse, G.; Furthmüller, J., Efficient Iterative Schemes for Ab Initio Total-Energy Calculations using a Plane-Wave Basis Set. Phys. Rev. B 1996, 54 (16), 11169-11186.

(52) Kresse, G.; Joubert, D., From Ultrasoft Pseudopotentials to the Projector Augmented-Wave Method. Phys. Rev. B 1999, 59 (3), 1758-1775.

(53) Perdew, J. P.; Ruzsinszky, A.; Csonka, G. I.; Vydrov, O. A.; Scuseria, G. E.; Constantin, L. A.; Zhou, X.; Burke, K., Restoring the Density-Gradient Expansion for Exchange in Solids and Surfaces. Phys. Rev. Lett. 2008, 100 (13), 136406.

(54) Hinuma, Y.; Hayashi, H.; Kumagai, Y.; Tanaka, I.; Oba, F., Comparison of Approximations in Density Functional Theory Calculations: Energetics and Structure of Binary Oxides. Phys. Rev. B 2017, 96 (9), 094102.

(55) Hinuma, Y.; Toyao, T.; Kamachi, T.; Maeno, Z.; Takakusagi, S.; Furukawa, S.; Takigawa, I.; Shimizu, K., Density Functional Theory Calculations of Oxygen Vacancy Formation and Subsequent Molecular Adsorption on Oxide Surfaces. J. Phys. Chem. C 2018, 122, 29435-29444. 
(56) Grimme, S.; Antony, J.; Ehrlich, S.; Krieg, H., A Consistent and Accurate Ab Initio Parametrization of Density Functional Dispersion Correction (DFT-D) for the 94 Elements H-Pu. J. Chem. Phys. 2010, 132, 154104.

(57) Grimme, S.; Ehrlich, S.; Goerigk, L., Effect of the Damping Function in Dispersion Corrected Density Functional Theory. J. Comput. Chem. 2011, 32, 1456-1465.

(58) Monkhorst, H. J.; Pack, J. D., Special Points for Brillouin-Zone Integrations. Phys. Rev. B. 1976, 13, 5188-5192.

(59) Kakekhani, A.; Roling, L. T.; Kulkarni, A.; Latimer, A. A.; Abroshan, H.; Schumann, J.; AlJama, H.; Siahrostami, S.; Ismail-Beigi, S.; Abild-Pedersen, F. et al. Nature of Lone-Pair-Surface Bonds and Their Scaling Reactions. Inorg. Chem. 2018, 57, $7222-7238$.

(60) Gaussian 09; Gaussian, Inc.: Wallingford, CT, 2009.

(61) Krishnan, R.; Binkley, J. S.; Seeger, R.; Pople, J. A. Self-Consistent Molecular Orbital Methods. XX. A Basis Set for Correlated Wave Functions. J. Chem. Phys., 1980, $72,650-654$.

(62) Yoshizawa, K. Nonradical Mechanism for Methane Hydroxylation by Iron-Oxo Complexes. Acc. Chem. Res. 2006, 39, 375-382.

(63) Tillotson, M. J.; Brett, P. M.; Bennett, R. A.; Grau-Crespo, R. Adsorption of Organic Molecules at the $\mathrm{TiO}_{2}(110)$ surface: The Effect of van der Waals Interactions. Surf. Sci. 2015, 632, 142-153. 
(64) Liu, L. V.; Tian, W. Q.; Chen, Y. K.; Zhang, Y. A.; Wang, Y. A. Theoretical Studies on Structures, ${ }^{13} \mathrm{C}$ NMR Chemical Shifts, Aromaticity, and Chemical Reactivity of Finite-Length Open-Ended Armchair Single-Walled Carbon Nanotubes. Nanoscale 2010, 2, 254-261.

(65) Ramamoorthy, M.; Vanderbilt, D.; King-Smith, R. D. First-Principles Calculations of the Energetics of Stoichiometric $\mathrm{TiO}_{2}$ Surfaces. Phys. Rev. B. 1994, 49, 16721-16727.

(66) Tsuji, Y.; Yoshizawa, K. Adsorption and Activation of Methane on the (110) Surface of Rutile-Type Metal Dioxides. J. Phys. Chem. C 2018, 122, 15359-15381.

(67) Rafique, M.; Shuai, Y.; Hassan, M. Structural, Electronic and Optical Properties of CO Adsorbed on the Defective Anatase $\mathrm{TiO}_{2}$ (101) Surface; a DFT Study. J. Mol. Struct. 2017, 1142, 11-17.

(68) Xu, M.; Shao, S.; Gao, B.; Lv, J.; Li, Q.; Wang, Y.; Wang, H.; Zhang, L.; Ma, Y. Anatase (101)-like Structural Model Revealed for Metastable Rutile $\mathrm{TiO}_{2}(011)$ Surface. ACS Appl. Mater. Interfaces 2017, 9, 7891-7896.

(69) Albright, T. A.; Burdett, J. K.; Whangbo, M.-H. Orbital Interactions in Chemistry, 2nd ed.; Wiley: Hoboken, 2013.

(70) Kode srl, Dragon (software for molecular descriptor calculation) version 7.0.6, 2016, https://chm.kode-solutions.net 
(71) Barone, P. M. V. B.; Camilo, Jr. A.; Galvão, D. S. Theoretical Approach to Identify Carcinogenic Activity of Polycyclic Aromatic Hydrocarbons. Phys. Rev. Lett. 1996, 77, 1186-1189.

(72) Braga, S. F.; Galvão, D. S. A Structure-Activity Study of Taxol, Taxotere, and Derivatives Using the Electronic Indices Methodology (EIM). J. Chem. Inf. Comput. Sci. 2003, 43, 699-706.

(73) Aptula, A. O.; Roberts, D. W.; Cronin, M. T. D. From Experiment to Theory: Molecular Orbital Parameters to Interpret the Skin Sensitization Potential of 5-Chloro-2-methylisothiazol-3-one and 2-Methylisothiazol-3-one. Chem. Res. Toxicol. 2005, 18, 324-329.

(74) Geurts, P.; Ernst, D.; Wehenkel, L. Extremely Randomized Trees. Mach. Learn. 2006, 63, 3-42.

(75) Hageman, J. A.; Westerhuis, J. A.; Frühauf, H.-W.; Rothenberg, G. Design and Assembly of Virtual Homogeneous Catalyst Libraries - Towards in Silico Catalyst Optimisation. Adv. Synth. Catal. 2006, 348, 361-369.

(76) Seko, A.; Tomoya, M.; Tsuda, K.; Tanaka, I. Machine Learning with Systematic Density-Functional Theory Calculations: Application to Melting Temperatures of Single- and Binary-Component Solids. Phys. Rev. B. 2014, 89, 054303. 
TOC Graphic

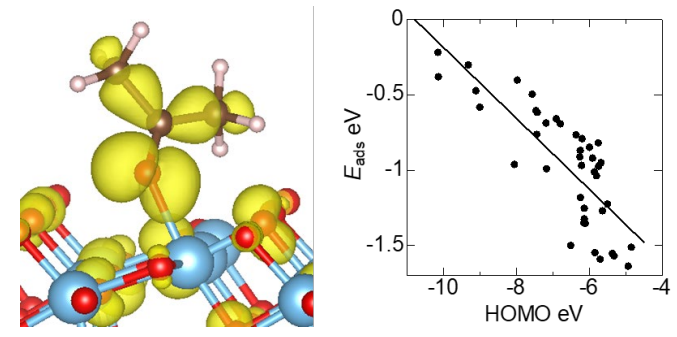

